\title{
Challenges in the treatment of dangerous and severe personality disorder ${ }^{\dagger}$
}

\author{
Kevin Howells, Gopi Krishnan \& Michael Daffern
}

\begin{abstract}
Dangerous and severe personality disorder (DSPD) services have a relatively short history but are increasingly involved in the implementation of therapeutic programmes. We describe the background to the DSPD initiative in England and consider issues that arise in planning and delivering treatment services. Two bodies of evidence are particularly relevant: previous research into personality disorder and its treatment, which we suggest is as yet of limited value, and research into the outcomes of offender treatment programmes. The latter is clearly relevant but greater consideration of adapting programmes for the patient population and of breadth of treatment is required in the DSPD setting. The important task is to integrate components for the treatment of personality disorder and offending behaviour in a holistic manner. We describe three further challenges in delivering treatment and suggest that ongoing evaluation of treatments is critical in this area of practice, given the impoverished knowledge base.
\end{abstract}

Personality disorder has long been the Cinderella of Cinderella health services. Many psychiatrists have considered personality disorder to be untreatable, some doubting the legitimacy of treating it within the health system. The issue of how to treat and manage (predominantly) men with severe personality or psychopathic disorder who also pose a risk to the public has been even more contentious. This latter group has traditionally been managed within the criminal justice system, but the problem of defining which agencies should be responsible for the management of individuals with personality disorders who pose a risk to the public was brought into sharp focus during the acrimonious public debate following the conviction of Michael Stone for the murder of a mother and child in 1998. Although Stone was considered to have a personality disorder, psychiatrists dealing with him did not consider the disorder treatable and hence he was not detainable under the Mental Health Act 1983. In February 1999 the UK government announced proposals to introduce the Dangerous and Severe Personality Disorder (DSPD) pilot programme,,$^{\ddagger}$ the legal provision for which would be embedded within a new legislative framework.

The DSPD programme was originally described as 'The Third Way' to deal with a group of individuals who, over many years, were at the boundary between the health and criminal justice systems. Four sites in high security (two in health settings - Rampton and Broadmoor Hospitals - and two in the prison estate - HMP Frankland and HMP Whitemoor) have been established and are in varying degrees of completion. A number of sites in medium security and community settings are in the process of being established. These units have been set up to pilot the provision of DSPD services in England, with the objectives of developing an evidence base with regard to this hitherto neglected population, researching effective treatments and thus potentially contributing to improved public protection.

It must be emphasised that DSPD is not a clinical category or classification. The working definition of DSPD (Department of Health et al, 2004) is a determination that:

- an individual presents a significant risk (of serious physical or psychological harm from which it would be difficult or impossible for the victim to recover)

- an individual presents with a significant disorder of personality

- the risk presented is functionally linked to the personality disorder (see discussion below).

${ }^{\dagger}$ For a commentary on this article see pp. 333-335, this issue.

${ }^{\ddagger}$ For an outline of the DSPD pilot programme see Feeney, A. (2003) Dangerous severe personality disorder. Advances in Psychiatric Treatment, 9, 349-358. Ed.

Kevin Howells is Professor of Forensic Clinical Psychology at Nottingham University (Division of Psychiatry, Nottingham University, Duncan MacMillan House, Porchester Road, Nottingham NG36AA, UK. Email: kevin.howells@nottingham.ac.uk) and Head of the Peaks Academic and Research Unit in the Nottinghamshire Healthcare Trust. Gopi Krishnan is Consultant Forensic Psychiatrist and Clinical Director of Personality Disorder Services at Rampton Hospital. Michael Daffern is Research Fellow in the Peaks Academic and Research Unit in the Nottinghamshire Healthcare Trust and Honorary Research Fellow in the Division of Psychiatry at Nottingham University. 


\section{Box 1 Criteria for admission to a DSPD unit}

- Ascore of 30 or above on the Revised Psychopathy Checklist (PCL-R; Hare, 1991) or

- A PCL-R score of 25-29 plus at least one DSM-IV personality disorder diagnosis other than antisocial personality disorder or

- Two or more DSM-IV personality disorder diagnoses

The criteria for admission to a DSPD unit are listed in Box 1.

There is a wide range of theoretical approaches for understanding personality disorder and its causation, and a correspondingly wide range of potential therapies. Psychodynamic (including psychoanalytic), behavioural, cognitive, interpersonal and social theories have all been advanced (for an overview see Alwin et al, 2006). How personality disorder is to be treated will depend on how it is defined and understood. Personality disorders are increasingly seen as statistical extremes in the distribution of normal personality traits (the continuum model), but they are also identified in terms of individual dysfunction. Dysfunction has been viewed as arising from impairment of the organisational, integrative and self-regulatory processes required to achieve evolutionary tasks of: (a) stability of the self system; (b) satisfactory interpersonal functioning (for example, meeting needs for intimacy, affiliation and attachment); and (c) social integration in the form of prosocial and cooperative behaviour (Livesley, 2003, 2007). Personality dysfunction and statistical deviation from the norm may be independent dimensions. Depending on the situation and the role of the person, personality may be highly abnormal in the statistical sense without being dysfunctional (Alwin et al, 2006).

Prevalence studies indicate that personality disorders are very common in adult prisoners, juveniles in prison and in mentally disordered offenders, with antisocial personality disorder being the most common category (Fazel \& Danesh, 2002). As yet we have little published data on the prevalence of the criteria defining DSPD in the prison population. There is evidence that offenders with personality disorders have a higher risk for violent crime (Powis, 2002), particularly when the disorder is of a psychopathic type (Hemphill et al, 1998). Evidence linking personality disorder to violent offending, including sexual offending, is crucial, given the requirement for DSPD admission that there be a functional link.
It is not at all clear how the functional link can be determined for the individual offender/patient, beyond determining that the person belongs to two populations that overlap, namely those with personality disorders and those who engage in serious violent offending. Demonstrating the presence of a functional link would require one of two sorts of clinical evidence: either evidence that manipulation (for example treatment) of personality disorder led to a reduction in violent offending or evidence that for the individual the two phenomena systematically covary over time in a way that suggests functional causation, that is, that periods of personality disorder are followed by (cause) periods in which offending is elevated. As we shall discuss below, evidence that treatment for personality disorder will reduce offending is not yet available. Indeed, one of the major purposes of the DSPD initiative is to determine whether such reduction is possible. The second (covariation) type of evidence is also not obtainable, for the reason that, by definition, personality disorders are stable aspects of the individual not expected to show marked temporal variation. Whereas it would be possible to demonstrate a functional link by temporal covariation between, say, substance misuse and offending, it is not possible for personality disorder. Substance misuse is typically episodic, and periods of misuse could be compared with periods of no use to determine whether criminal behaviour is lower in the latter. If personality disorders turn out to be less stable than has previously been assumed, then covariation evidence may indeed be useful.

\section{Assessment}

There is a consensus that structured assessments are required for both the personality disorder component (Tyrer et al, 2007) and the risk/offending behaviour component of DSPD. All individuals admitted to the four high secure DSPD services complete the structured assessments listed in Box 2. These assessments relate more to the presence or absence of risk and personality disorder (admission criteria) than to the assessment of clinical therapeutic needs. Thus, they would need to be - and are - followed by more detailed clinical assessments that allow for a full formulation of the patient's problems, both clinical and criminogenic, and for subsequent identification of specific treatment targets. Such assessments need to cover behavioural, cognitive, affective, interpersonal and self-regulatory domains. The crucial role of case formulation in this context has been emphasised in a recent report (Alwin et al, 2006: p. 32):

'The provision of effective treatment for individuals with personality disorder requires the ability to place 
Box 2 Minimum assessments required on admission to a DSPD unit

Risk assessments

Violence:

- Violence Risk Scale (VRS; Wong \& Gordon, 1999-2003)

- Historical, Clinical and Risk Management (HCR-20) scale (Webster et al, 1997)

Sexual offending:

- Risk Matrix 2000 (Thornton et al, 2003)

- Static 99 (Nunes et al, 2002)

- Structured Assessment of Risk and Need (SARN; Thornton, 2002)

Personality disorder

- The Revised Psychopathy Checklist (PCLR; Hare, 1991)

- Psychopathy Checklist - Screening Version V (PCL-SV; Hart et al, 1995)

- International Personality Disorder Examination (IPDE; World Health Organization, 1997)

their experiences in a contextual and explanatory framework that can help to raise that person's own awareness of their behaviours, thoughts and emotions ... Formulation is necessary ... (and) ... goes beyond diagnosis through the generation of a working model based on an assessment of the range of personality traits presented'.

\section{Treatment of personality disorders}

People with personality disorders have received a wide range of treatments, including pharmacological, psychoanalytic, cognitive, cognitive analytic, dialectical behaviour, community and other therapies (Box 3). Although the evidence base is not large, there are indications in the literature

\section{Box 3 Examples of therapies for personality} disorders

- Dialectical behaviour therapy

- Cognitive-behavioural therapy (including schema therapy)

- Emotional regulation therapies (e.g. anger management)

- Cognitive analytic therapy

- Therapeutic communities

- Livesley's eclectic/pragmatic approach (see text) that some psychotherapeutic interventions may be effective (Bateman \& Fonagy, 1999; Perry et al, 1999; Leichsenring \& Leibing, 2003; Verheul et al, 2003; Bateman \& Tyrer, 2004), but it remains to be determined whether any one therapeutic approach is more effective than any other or whether it is the non-specific aspects of a treatment (for example, structure, specification of targets for change and forming a therapeutic relationship) that produce the treatment effect. Although all of these therapies have some evidential support in other mental disorders, and even in personality disorders, there is virtually no evidence of their effectiveness with individuals who combine severe personality disorder with high-risk forms of violence (Warren et al, 2003). The challenges for DSPD services are two-fold: to adapt such interventions for the distinctive characteristics of people with DSPD (see, as an example, the discussion of low treatment readiness below) and to prioritise research and evaluation into the effectiveness of these programmes in a DSPD context (also discussed below).

For the purposes of the present article, the literature cited above is of limited relevance, for two reasons. First, previous outcome studies have looked at a restricted set of personality disorders, most commonly borderline personality disorder. It is not yet known whether offenders with borderline personality disorder will form a large proportion of those admitted to DSPD units. Given the emphasis on psychopathy in DSPD admission criteria, it is likely that antisocial personality disorder will predominate, with other DSM-IV (American Psychiatric Association, 1994) Cluster B disorders, including borderline personality disorder, also evident. The therapy outcome data for antisocial personality disorder and psychopathic disorder are thin indeed (D'Silva et al, 2004), with some evidence of adverse treatment outcomes (Harris et al, 1994).

Second, even when outcome studies do exist they rarely address the needs of forensic or high-risk populations of a DSPD type, who are likely to reject rather than seek treatment (Tyrer et al, 2003) and to manifest their disorder in particularly antisocial ways. At present, it is reasonable to conclude that we do not know which, if any, treatments for personality disorder will prove to be effective for DSPD (Warren et al, 2003), but that some interventions (for example, dialectical behaviour therapy, cognitivebehavioural therapy, psychodynamic therapy) have sufficient initial credibility in related populations to warrant implementing exploratory therapeutic programmes, which should then be evaluated as to their effectiveness (see below).

Livesley $(2003,2007)$ has argued for an integrated and multifaceted approach to treating personality disorder in people with DSPD. Integration has three 
components: an eclectic use of diverse models and therapeutic strategies, based on their demonstrated effectiveness; delivering treatments in an integrated way; and focusing the treatment efforts to produce integration and coherence of personality functioning. Within Livesley's model, the breakdown of integrative functioning and of a coherent sense of identity is central to personality disorders. Livesley's approach might be characterised as pragmatic eclecticism, selecting different treatments to target specific components of personality disorder while also addressing the overall integration of the components themselves. Integration of the different treatments is achieved through non-specific aspects, with the therapeutic alliance, promotion of motivation and the structured nature of the treatments being particularly important. It will be apparent from this brief account of Livesley's model that achieving integration will be even more important, and challenging, in a DSPD context, given that offending behaviour will also require integration within the general treatment framework. If his model is correct, it would also suggest that separate therapeutic programmes typical of current correctional offender rehabilitation (see below) would be deficient in a DSPD context.

\section{Treatment of high-risk offending behaviours}

There are some fascinating contrasts to be made between therapeutic approaches to personality disorder and therapeutic approaches to high-risk offending behaviours. As already mentioned, therapies for personality disorder have had a wide range of theoretical orientations, with no one orientation yet demonstrated to be superior. They are more likely to be delivered in mental health settings (although two of the four DSPD units in England and Wales are based in prisons). There appears as yet to be little consensus among researchers and clinicians as to what types of programme and programme features for personality disorder are likely to be associated with good outcomes.

The development of offending behaviour programmes, on the other hand, has created what appears to be a current consensus, based on the 'What Works' literature (McGuire, 1995, 2002), that cognitive-behavioural treatments are the most effective, and such treatments and programmes dominate the offender treatment and rehabilitation scene internationally. The variation between offending behaviour programmes is more one of type of offending or type of criminogenic need being targeted (sex offending, violent behaviour, anger problems, cognitive and problem-solving deficits, substance misuse) rather than of fundamental theoretical
Box 4 'What works' principles identified in the literature on offender programmes

Risk principle: the intensity of the treatment offered should be proportional to the risk of future serious offending

Needs principle: effective treatment targets criminogenic needs, that is, factors shown to predict future offending

Responsivity principle: the manner of delivery of the programme should be consistent with the characteristics and abilities of the group being treated

Professional discretion: professional override is necessary in some treatment decisions

Programme integrity: the programme should be conducted in practice as intended in its theory and design

orientation. In the main, offender treatment theorists and clinicians are confident that programme features associated with good outcomes have been identified, including the principles of risk, need, responsivity, professional discretion and programme integrity (Andrews \& Bonta, 2003), and the task has become one of ensuring that such principles do govern programme design and implementation (Box 4).

Such principles have only recently begun to influence forensic mental health services (Howells et al, 2004), where a different culture exists, apparently uncomfortable with and even inimical to 'correctional' ways of working. Arguably, DSPD is the area of forensic mental health services in which most progress has been made in building the principles of offending behaviour programmes into the service, even though integrated treatment approaches are still at an early stage of development. Certainly, risk (Box 2) and needs assessment are a major component of the assessment programme in DSPD services, just as they are within correctional programmes. It should be noted, however, that the risk principle in offender rehabilitation relates to 'dosage' of the treatment, that is, ensuring that those of higher risk receive more intensive treatment. Individuals admitted to DSPD services are already shown to pose a high risk; therefore the focus of risk assessment must be the identification of dynamic risk factors that should be addressed as treatment targets.

The challenge of treating people with DSPD is that both a personality disorder and an offending behaviour focus are required. Individuals need to change dysfunctional aspects of their personality 
but also to change the dynamic (criminogenic) needs that lead them to offend. Addressing both personality disorder and offending behaviour raises serious issues of how the different theoretical models underlying personality disorder and offending can be made compatible, for the benefit of both staff and the offender/patient. How, for example, could a psychodynamic view of personality disorder be married with a cognitive-behavioural view of offending behaviour?

Offending behaviour treatments in DSPD units should, in principle, focus on the same criminogenic needs as would programmes with any high-risk offenders in the criminal justice system. Such programmes would typically address sexual offending, anger and violence, substance misuse, and cognitive skills and problem-solving. The Chromis programme (HM Prison Service, 2005) and the Violence Reduction Program (Wong, 2004) are two credible and sophisticated programmes of this sort undergoing trial within DSPD services.

The exact nature of the previous offences committed by people admitted to DSPD units is yet to be analysed but it is likely that severe sexual and violent offending will prove to be common, both as index offences and as previous behaviour. In planning treatments for such offending it would be foolish to ignore the considerable scientific literature that now exists in relation to understanding and treating these forms of behaviour (Marshall et al, 1999; Polaschek \& Collie, 2004). A sceptic might make the rejoinder that such knowledge and treatments are based on 'mentally normal' offenders and are hence not relevant to mentally disordered offenders, particularly those with personality disorders. This argument is unconvincing for several reasons. First, it is probable that many of the thousands of offenders who have been treated in such programmes would indeed meet the criteria for personality disorder were they to be assessed. We know that personality disorders are very common in offender groups but that formal screening for such disorders is rare. Second, what evidence is available suggests that the criminogenic needs (dynamic risk factors) of 'mentally normal' and mentally disordered offenders are largely the same (Bonta et al, 1998). This provides a prima facie case for delivering offender programmes for sexual and violent offending (and criminogenic programmes such as substance misuse and cognitive skills) to people with DSPD.

The essential requirement, of course, is that the responsivity principle is observed (Andrews \& Bonta, 2003), that is, that the style of delivery of these programmes, and to a degree their content, are modified to make them suitable for participants who have personality disorders, in the same way that offender programmes would have to be modified for delivery to those with intellectual disabilities or distinctive ethnic or cultural needs (Day, 2003). Understanding the difficulties that people with DSPD are likely to experience in completing offending behaviour programmes and then modifying the programmes to accommodate their needs are important tasks for the future.

Despite the dissemination of 'What Works' offender treatment programmes around the world, in recent years critiques have begun to emerge, which might suggest a need to broaden and refine the approach. Such critiques are very pertinent to the development of DSPD treatments in that the new approaches advocated require intensive, individually focused therapeutic efforts which are difficult to deliver in typical offender programmes based within the criminal justice system. The approaches are feasible within DSPD settings, as these generally have high levels of clinical resources and staffing and are designed for intensive and prolonged treatment. One such critique has been recently provided by Thomas-Peter $(2006 a, b)$, who points to the dangers within 'What Works' programmes of narrowness of therapeutic expertise, the fragmentation of knowledge and the 'disaggregation' of the individual into a series of unconnected problems. From this perspective, a more integrated and holistic approach to offender treatment is required.

Although such criticisms would not be accepted by all advocates of offender programmes, the problems of 'disaggregating' participants in therapy and the necessity to address offender needs as well as criminogenic needs in a holistic way is being increasingly recognised. Ward \& Brown's critique of the offender treatment literature from the 'good lives' perspective also points in the same direction (Ward \& Brown, 2004). The question of how both criminogenic and non-criminogenic programmes should be sequenced is an important one.

\section{Challenges in the implementation of treatment for DSPD}

It is common in new initiatives such as the DSPD programme to witness a gap between theories and expectations during planning and the harsh realities of the implementation. In this section we wish to address some of the challenges that inhabit the gap.

\section{The reluctant patient}

Low engagement in therapeutic programmes and non-completion are vital issues in the treatment and rehabilitation of offenders, of mentally disordered offenders and of patients admitted to DSPD services. The potential consequences of low engagement are 
many and include diminished staff morale, poor institutional support for programmes and poor treatment outcomes.

There have been many theoretical attempts to explain low engagement. Low motivation, resistance and low responsivity are terms sometimes used to explain it but all of these terms are problematic and in recent years we have seen the suggestion that such concepts be subsumed under the term (low) treatment readiness (Ward et al, 2004). For a review of the applicability of the concept of low treatment readiness to offenders with personality disorders see Howells \& Day (2007). Clinical observation suggests that offenders with personality disorders are very often 'unready' for treatment, in part because of their internal characteristics (beliefs, emotional reactions, identities and behavioural deficits that undermine engagement and the forming of a therapeutic alliance) and in part because of external, situational influences such as perceived coercion into treatment.

It follows that the assessment and modification (when low) of readiness should be a vital task in services for people with DSPD. Clinical evaluation of readiness is particularly relevant at three points: at the point of referral, when the patient/offender is being assessed for suitability for admission; shortly after admission, when there is a clinical need to understand the patient's perceptions of and expectations about treatment; and as part of treatment planning and monitoring.

\section{Maintaining staff morale and a positive therapeutic environment}

Preserving a cohesive and optimistic therapeutic environment can be challenged by the aggression, self-harm and sexually abusive behaviour that is sometimes shown by in-patients with psychopathic and personality disorders in DSPD and similar units. These behaviours have the potential to disrupt achievement of therapeutic objectives. There is the risk that perpetrators will be denied access to certain programmes or therapeutic activities or that other patients may be reluctant to attend programmes because of a fear of victimisation. Staff resources can be directed away from the provision of therapy to the management of disruptive patients. A tense and hostile therapeutic milieu is to be avoided because it is likely to distract patients from treatment tasks and to erode staff persistence, confidence and optimism about therapeutic programmes.

In DSPD units, structure is required to ensure that patients experience a sense of predictability about the environment and of safety and support while participating in the lengthy therapeutic process. The demands and stresses of living in long-term detention are many, even in the best-planned environments, particularly for patients who have limited resources for coping. Providing a structured and safe environment has to be balanced against ensuring it is not so contrived that therapeutic gains, if made, cannot be generalised to more normal environments. Thus, measurement and monitoring of the therapeutic environment is as important as conventional monitoring of the progress of the patient.

\section{'Does it work?'}

Three relatively independent types of evaluation are critical in relation to treatment programmes for DSPD: evaluation of regime, evaluation of specific component treatments and evaluation of programme quality. The first is predicated on the fact that DSPD services are more than the sum of particular treatment programmes. As discussed above, patients in DSPD units live in a structured and highly regulated institutional environment, in most cases for several years. The therapeutic climate of DSPD units is likely to vary, as are ways of relating to patients and managing their behaviour. The aggregate of all these general and specific programme factors constitutes the regime and it is important to establish whether that regime is more effective in bringing about change, both clinical and criminogenic, than another regime. An important evaluation, for example, although not one that would be easy to conduct, is whether forensic hospital DSPD regimes are more effective than prison-based ones. There would be major obstacles to randomisation of allocation to hospitals $v$. prisons. A regime evaluation would require measurement of the non-specific aspects of therapeutic environments such as the therapeutic climate (Campling et al, 2004), and would need to include important longer-term outcomes such as effective resettlement and recidivism reduction.

An evaluation of component treatments is a different matter. The evaluation question here is whether a particular treatment (for example, dialectical behaviour therapy) is more effective than no treatment or an alternative treatment. Given that patients with DSPD are likely to receive multiple, sequential interventions, it will be difficult to test whether any one treatment affects long-term outcomes such as resettlement and reconviction, as the study would be confounded by the other treatments received.

Randomised controlled trials (RCTs) are clearly relevant to three types of evaluation mentioned above. Although the importance of RCTs is widely supported, and their absence in relation to personality disorder, particularly personality disorder associated 


\section{Box 5 Single-case designs}

Thetraditionalbugbearsofsingle-casemethods have been, first, their perceived vulnerability to threats to internal validity, that is, to the possibility that the treatment effect observed in a particular patient (for example, improvement in control of aggression) may be attributable to factors other than the treatment itself; and second, they appear also to be vulnerable to a major threat to external validity in the form of uncertain generalisability of findings, namely from the individual to a larger group of patients. Both these potential weaknesses are addressed to some extent in contemporary versions of single-case methodology (Bloom et al, 2003).

with violent offending, widely lamented, the fact that RCTs are not the only methodology for gaining knowledge about treatment effectiveness is increasingly acknowledged (Bloom et al, 2003; Davies et al, 2007). Sophisticated methods now exist for controlled evaluations of single cases in the form of single-case designs (Bloom et al, 2003) (Box 5). Davies et al (2007) have suggested that the conditions prevailing in DSPD services are particularly suited to the requirements of single-case methods, particularly the need for prolonged pre-treatment baseline observation and for intensive observation through baseline, intervention and follow-up phases.

The third type of treatment evaluation is that of the quality of the programme. Quality control of treatment programmes - accreditation, programme checklists, integrity checks, etc. - is common in offender treatment programmes in criminal justice systems. In England and Wales, Canada and New Zealand for example, offender programmes must pass rigorous standards, typically in the form of accreditation requirements. Systematic evaluation of programmes against such criteria is less common in forensic mental health settings, for a variety of reasons (Howells et al, 2004). In part, the reason for the difference is likely to be that forensic mental health professionals, because of the limited knowledge base in the literature, are less confident as to what are the critical programme features associated with good outcomes, whereas offender programme professionals have a stronger sense of 'What Works' in offender rehabilitation (McGuire, 2002).

Evaluation of treatment is likely to remain a priority within DSPD treatment services. For a more detailed discussion of evaluation issues in DSPD, the reader should consult a review by Langton (2007).

\section{Conclusions}

The above challenges are only a few of those faced by mental health professionals and managers within DSPD services. Managing expectations of referring agencies, government departments and the broader community, and ensuring that staff with appropriate training and expertise can be recruited and retained are also formidable tasks.

\section{Declaration of interest}

None. The views expressed in this article are those of the authors and do not reflect the opinions of DSPD services, the Department of Health or the Home Office.

\section{References}

Alwin, N., Blackburn, R., Davidson, K., et al (2006) Understanding Personality Disorder. A Report by the British Psychological Society. British Psychological Society.

American Psychiatric Association (1994) Diagnostic and Statistical Manual of Mental Disorders (4th edn) (DSM-IV). APA.

Andrews, D. A. \& Bonta, J. (2003) Psychology of Criminal Conduct (3rd edn). Anderson Publishing.

Bateman, A. \& Fonagy, P. (1999) Effectiveness of partial hospitalization in the treatment of borderline personality disorder. A randomized controlled trial. American Journal of Psychiatry, 156, 1563-1569.

Bateman, A. W. \& Tyrer, P. (2004) Psychological treatments for personality disorders. Advances in Psychiatric Treatment, 10, 378-388.

Bloom, M., Fischer, J. \& Orme, J. G. (2003) Evaluating Practice: Guidelines for the Accountable Professional (4th edn). Allyn \& Bacon.

Bonta, J., Hanson, K. \& Law, M. (1998) The prediction of criminal and violent recidivism among mentally disordered offenders. A meta-analysis. Psychological Bulletin, 123, 123-142.

Campling, P., Davis, S. \& Farquharson, G. (eds) (2004) From Toxic Institutions to Therapeutic Environments: Residential Settings in Mental Health Services. Gaskell.

Davies, J., Howells, K. \& Jones, L. (2007) Evaluating innovative treatments in forensic mental health. A role for single-case methodology? Journal of Forensic Psychiatry and Psychology, in press.

Day, A. (2003) Reducing the risk of re-offending among Australian indigenous offenders. What works for whom? Journal of Offender Rehabilitation, 37(2), 1-16.

Department of Health, Home Office \& HM Prison Service (2004) Dangerous and Severe Personality Disorder (DSPD) High Security Services: Planning and Delivery Guide. Home Office.

D'Silva, K., Duggan. C. \& McCarthy, L. (2004) Does treatment really make psychopaths worse? A review of the evidence. Journal of Personality Disorders, 18, 163-177.

Fazel, S. \& Danesh, J. (2002) Serious mental disorder in 23000 prisoners. A systematic review of 62 surveys. Lancet, 359, 545-550.

Hare, R. (1991) Manual for the Hare Psychopathy Checklist-Revised. Multi-Health Systems.

Harris, G., Rice, M. \& Cormier, C. (1994) Psychopaths: is the therapeutic community therapeutic? Therapeutic Community, 15, 283-299.

Hart, S., Cox, D. \& Hare, R. (1995) The Hare PCL:SV Psychopathy Checklist: Screening Version. Multi-Health Systems.

Hemphill, J., Hare, R. \& Wong, S. (1998) Psychopathy and recidivism. A review. Legal and Criminological Psychology, 3, 139-170. 
HM Prison Service (2005) Chromis Manuals. Offending Behaviour Programme Unit.

Howells, K. \& Day, A. (2007) Readiness for treatment in high risk offenders with personality disorders. Psychology, Crime and Law, 13, 47-56.

Howells, K., Day, A. \& Thomas-Peter, B. (2004) Treating violence. Forensic mental health and criminological models compared. Journal of Forensic Psychiatry and Psychology, 15, 391-406.

Langton, C. M. (2007) Assessment implications of "What Works" research for Dangerous and Severe Personality Disorders service evaluations. Psychology, Crime and Law, 13, 97-112.

Leichsenring, F. \& Leibing, E. (2003) The effectiveness of psychodynamic therapy and cognitive behaviour therapy in the treatment of personality disorders. A meta-analysis. American Journal of Psychiatry, 160, 1223-1232.

Livesley, W. J. (2003) Diagnostic dilemmas in the classification of personality disorder. In Advancing DSM: Dilemmas in Psychiatric Diagnosis (eds K. Phillips, M. First \& H. A. Pincus), pp. 153-189. American Psychiatric Association Press.

Livesley, W. J. (2007) The relevance of an integrated approach to the treatment of personality disordered offenders. Psychology, Crime and Law, 13, 27-46.

Marshall, W. L., Anderson, D. \& Fernandez, Y. (1999) Cognitive Behavioural Treatment of Sex Offenders. John Wiley \& Sons.

McGuire, J. (ed.) (1995) What Works: Reducing Reoffending. John Wiley \& Sons.

McGuire, J. (ed.) (2002) Offender Rehabilitation and Treatment: Effective Programmes and Policies to Reduce Re-offending. John Wiley \& Sons.

Nunes, K. L., Firestone, P., Bradford, J. M., et al (2002) A comparison of modified versions of the Static-99 and the Sex Offender Risk Appraisal Guide (SORAG). Sexual Abuse: A Journal of Research and Treatment, 14, 253-269.

Perry, J. C., Banon, E. \& Ianni, F. (1999) Effectiveness of psychotherapy for personality disorders. American Journal of Psychiatry, 156, 1312-1321.

Polaschek, D. L. L. \& Collie, R. M. (2004) Rehabilitating serious violent adult offenders. An empirical and theoretical stocktake. Psychology, Crime and Law, 3, 321-334.

Powis, B. (2002) Offenders' Risk of Serious Harm. A Literature Review (Occasional Paper no. 81). Home Office.

Thomas-Peter, B.A. (2006a) The modern context of psychology in corrections: influences, limitations and values of "What Works". In Psychology Research in Prisons (ed. G. Towl), pp. 24-39. Blackwell.

Thomas-Peter, B.A. (2006b) The needs of offenders and the process of changing them. In Psychology Research in Prisons (ed. G. Towl), pp. 40-53. Blackwell.

Thornton, D. (2002) Constructing and testing a framework for dynamic risk assessment. Sexual Abuse: A Journal of Research and Treatment, 14, 139-153.

Thornton, D., Mann, R., Webster, S., et al (2003) Understanding and managing sexually coercive behaviour. Annals of the New York Academy of Science, 989, 225-235.

Tyrer, P., Mitchard, S., Methuen, C., et al (2003) Treatment rejecting and treatment seeking personality disorders: Type $\mathrm{R}$ and Type S. Journal of Personality Disorders, 17, 263-268.

Tyrer, P., Coombs, N., Ibrahimi, F., et al (2007) Critical developments in the assessment of personality disorder. British Journal of Psychiatry, 190 (suppl. 49), s51-s59.

Verheul, R., Van Den Bosch, L. M. C., Koeter, M. W. J., et al (2003) Dialectical behaviour therapy for women with borderline personality disorder. 12-month, randomised clinical trial in The Netherlands. British Journal of Psychiatry, 182, 135-140.

Ward, T. \& Brown, M. (2004) The good lives model and conceptual issues in offender rehabilitation. Psychology, Crime and Law, 3, 243-257.

Ward, T., Day, A., Howells, K. et al (2004) The multifactor offender readiness model. Aggression and Violent Behavior, 9, 645-673.

Warren, F., Preedy-Fayers, K., McGauley, G., et al (2003) Review of Treatments for Severe Personality Disorder. Home Office.

Webster, C. D., Douglas K. S., Eaves, D., et al (1997) HCR-20: Assessing Risk for Violence (Version 2). Simon Fraser University, Forensic Psychiatric Services Commission of British Columbia.
Wong, S. (2004) The Violence Reduction Program. Correctional Services Canada.

Wong, S. C. P. \& Gordon, A. (1999-2003) Violence Risk Scale. Department of Psychology, University of Saskatchewan. http:/ / www.psynergy.ca

World Health Organization (1997) International Personality Disorder Examination (IPDE Manual). American Psychiatric Publishing.

\section{MCQs}

1 The four high-security DSPD sites:

a are all based in the prison system

$\mathrm{b}$ are all based in the National Health Service

c are divided between the prison system and the National Health Service

$\mathrm{d}$ have not yet been located within a service

e are not based in purpose-built accommodation.

2 The responsivity principle in offender treatment refers to:

a whether or not the person is responsive to treatment

$\mathrm{b}$ the level of engagement in treatment

c warmth and acceptance on the part of clinicians

$\mathrm{d}$ the treatability of the person

e adapting treatment for the particular characteristics of the group being treated.

3 Treatment for DSPD should:

a focus mainly on non-criminogenic needs

b focus mainly on criminogenic needs

c be mainly concerned with treating the personality disorder

$\mathrm{d}$ address both criminogenic and non-criminogenic needs

e focus on behavioural management rather than treatment.

4 Evaluation of treatment effectiveness for DSPD:

a must await a definitive RCT

$\mathrm{b}$ is largely unnecessary as we know what works

c is impossible given the complexity of cases

d should include single-case methodologies

e can only be qualitative rather than quantitative.

\section{MCQ answers}

$\begin{array}{llllll}1 & & \text { 2 } & & 3 & \\ \text { a F } & \text { a F } & \text { a F } & \text { a F } \\ \text { b F } & \text { b F } & \text { b F } & \text { b F } \\ \text { c T } & \text { c F } & \text { c F } & \text { c F } \\ \text { d F } & \text { d F } & \text { d T } & \text { d T } \\ \text { e F } & \text { e T } & \text { e F } & \text { e F }\end{array}$

